\title{
Autonomie et contrôle du travail enseignant
}

Une enquête dans les pays européens

Autonomy and inspection of teaching work. A European survey

Autonomía y control del trabajo del docente. Una encuesta en los países europeos

\section{Nathalie Mons}

\section{OpenEdition}

\section{Journals}

Édition électronique

URL : http://journals.openedition.org/ries/449

DOI : 10.4000/ries.449

ISSN : 2261-4265

\section{Éditeur}

Centre international d'études pédagogiques

\section{Édition imprimée}

Date de publication : 1 septembre 2008

Pagination : 109-117

ISBN : 978-2-85420-573-2

ISSN : $1254-4590$

Référence électronique

Nathalie Mons, "Autonomie et contrôle du travail enseignant », Revue internationale d'éducation de Sèvres [En ligne], 48 | septembre 2008, mis en ligne le 28 juin 2011, consulté le 26 avril 2021. URL http://journals.openedition.org/ries/449 ; DOl : https://doi.org/10.4000/ries.449 


\section{Autonomie et contrôle du travail enseignant}

\section{Une enquête dans les pays européens}

\section{Nathalie Mons}

Les deux dernières décennies ont été marquées, dans la très grande majorité des pays européens, par une extension des attributions confiées aux enseignants, dans un nouveau contexte d'autonomie scolaire : participation à la conception des contenus d'enseignement grâce à l'adaptation locale des programmes scolaires, nouvelles missions de remplacement des collègues absents ou d'encadrement des enseignants débutants, participation à des activités de formation continue... (Maroy, 2005; Eurydice, 2008). Ainsi, aux côtés des prérogatives qui ont traditionnellement constitué le cœur du métier d'enseignant depuis la création au XIX ${ }^{\mathrm{e}}$ siècle de la forme scolaire classique, se sont développées de nouvelles missions qui renouvellent le regard porté sur cette activité professionnelle. Au-delà des murs de sa classe, au-delà des interactions qu'il entretient au quotidien avec ses élèves, l'enseignant doit de plus en plus participer à des activités pédagogiques qui se développent au sein d'un établissement scolaire devenu plus autonome.

Cet élargissement des missions des enseignants dans le cadre d'un contexte d'autonomie a-t-il conduit, comme l'affirme la théorie de l' "Étatévaluateur»(Neave, 1988; Broadfoot, 2000; Maroy, 2005), à un accroissement de leur responsabilisation? Y a-t-il un lien entre l'accroissement des attributions et la fréquence des contrôles? L'intensité de l'autonomie dont bénéficient les enseignants est-elle corrélée à certains mécanismes d'évaluation? L'expansion des responsabilités est-elle associée au développement d'incitants récompensant les enseignants de leur engagement dans ces nouvelles tâches?

Selon la théorie de l'État-évaluateur, un nouveau modèle de gouvernance par les résultats aurait accompagné l'autonomie croissante des acteurs de terrain, autonomie qui résulte de réformes multiples ayant radicalement modifié le mode de fonctionnement des systèmes scolaires depuis près de trois décennies (flexibilité nouvelle dans les curricula, développement de nouvelles structures scolaires privées, libre choix de l'école...). Si les acteurs de terrain (les enseignants...) et les entités locales (les établissements scolaires ou les autorités déconcentrées en charge de l'éducation) bénéficient de marges de manœuvre plus importantes dans les choix de mise en œuvre des politiques éducatives désormais contextualisées, ce nouveau mode de gouvernance se caractérise aussi 
par la continuité d'un État central ${ }^{1}$ organisateur fort en charge de la définition des objectifs du système scolaire, par l'élaboration de «contrats » plus ou moins implicites entre ce centre et les entités locales et par la création d'un système d'évaluations externes et d'auto-évaluations associé à une panoplie d'incitants et de sanctions symboliques ou matériels. Plus spécifiquement, ce modèle est associé à une « défiance accrue à l'égard de l'autonomie professionnelle du corps enseignant, si elle n'est pas «encadrée» par de nouveaux systèmes d'évaluation de leurs pratiques et de leurs résultats. La confiance dans le professionnalisme enseignant se lézarde»(Maroy, 2005). Avec le système de l'État-évaluateur, nous sommes donc dans une situation a priori paradoxale caractérisée à la fois par un élargissement de l'autonomie et des responsabilités des enseignants et par un accroissement des contrôles à distance qui ne s'effectuent plus sur la conformité à la règle mais sur l'atteinte des objectifs assignés. Mais le paradoxe n'est qu'apparent, il révèle qu'autonomie ne signifie pas nouvelles libertés acquises. L'État-évaluateur n'est pas totalement en rupture avec l'organisation bureaucratique traditionnelle, il offre seulement un nouveau mode de régulation fondé sur les résultats.

Pour vérifier le bien-fondé de cette théorie, nous avons conduit une recherche au sein des pays européens, dans le cadre de l'agence Eurydice rattachée à la Commission Européenne (Eurydice, 2008). Une grille de contenu, concernant les responsabilités, l'autonomie et l'évaluation des enseignants a été soumise à l'ensemble des vingt-neuf unités nationales du réseau Eurydice. Ces questionnements étaient mis en perspective dans un cadre historique (pour mieux comprendre les facteurs expliquant la mise en œuvre de ces nouvelles politiques) et institutionnel (acteurs en charge des responsabilités, instruments légaux utilisés...). L'enquête s'est concentrée sur l'analyse de l'enseignement primaire et secondaire inférieur (CITE 1 et 2). Tous les pays du réseau Eurydice, à l'exception de la Turquie, ont pu être couverts par l'étude ${ }^{2}$.

L'enquête a révélé qu'il n'existait pas de simultanéité entre le développement de l'autonomie des enseignants, l'élargissement des tâches qui leur incombent et l'accroissement de leur évaluation. Cette absence de lien chronologique s'explique par le fait que l'ensemble de ces mutations ont rarement été intégrées dans un cadre institutionnel global cohérent. Ainsi, l'élargissement du périmètre d'activités des enseignants n'a pas souvent été le fruit d'une analyse structurée des spécificités du métier de ces professionnels. Dans la très grande majorité des pays européens, des législations diverses - celles relatives à la conception des programmes scolaires, aux conditions de service, à la formation

1. Il peut s'agir de l'État central ou des autorités supérieures en charge de l'éducation (les communautés autonomes en Espagne, les Länder en Allemagne, les cantons en Suisse...).

2. Allemagne, Autriche, Belgique, Chypre, Danemark, Espagne, Estonie, Finlande, France, Grèce, Hongrie, Irlande, Islande, Italie, Lettonie, Liechtenstein, Lituanie, Luxembourg, Malte, Norvège, Pays-Bas, Pologne, Portugal, République tchèque, Royaume-Uni, Slovaquie, Slovénie, Suède. 
continue... - se sont empilées progressivement pour conduire à un renouvellement pas à pas de l'activité enseignante. Aussi, le plus souvent, cet élargissement progressif des prérogatives dans un nouveau cadre d'autonomie n'a-t-il pas été accompagné par un mouvement mécanique et parallèle de responsabilisation et de contrôle structurés. Les mécanismes d'évaluation mais aussi d'incitation apparaissent chronologiquement décalés dans leur mise en œuvre par rapport à l'accroissement des responsabilités des enseignants.

Pour autant, si le lien n'a pas été automatique entre l'attribution de nouvelles compétences et leurs évaluations, un accroissement du contrôle du travail enseignant se dessine progressivement sur les deux dernières décennies. Cette responsabilisation présente quatre principales caractéristiques. Elle revêt tout d'abord des formes variées, depuis l'inspection externe individuelle traditionnelle axée sur les processus jusqu'à l'auto-évaluation de l'école incluant une analyse du travail enseignant, en passant par des entretiens d'évaluation menés en interne par le chef d'établissement. Ces évaluations sont marquées - seconde caractéristique - par des analyses en termes de résultats qui contrastent avec les traditionnels contrôles fondés sur l'observation des processus. Troisième trait : l'évaluation actuelle oscille entre dimension collective et individuelle : si les années 1990 avaient été marquées par l'élargissement de l'autonomie scolaire et le développement d'une responsabilisation de l'équipe enseignante associée à de faibles conséquences pour les acteurs, depuis le milieu de la décennie 2000, on assiste à un renforcement des mécanismes de redevabilité individuels à forts enjeux. Enfin, cet élargissement des attributions des enseignants et le développement de mécanismes de responsabilisation n'ont pas, dans la majorité des pays, été associés au développement en miroir de mesures d'incitation.

\section{RESPONSABILISATION DES ENSEIGNANTS : DES MODALITÉS D'ÉVALUATION VARIÉES}

Parce que le travail enseignant est compris aujourd'hui dans une logique à la fois individuelle - le travail du professionnel - et collective - les résultats de l'équipe pédagogique d'un établissement scolaire -, les modalités de contrôle de cette activité sont désormais multiples.

De nombreux pays sont tout d'abord restés attachés à l'inspection des enseignants conduite par un corps de spécialistes externes à l'établissement. Ces inspectorats peuvent être rattachés aux autorités nationales, comme en République tchèque et en France, ou sous la tutelle des autorités locales en charge de l'éducation comme en Espagne, en Allemagne ou en Autriche. Si ces inspections existent traditionnellement dans les systèmes scolaires, la période actuelle semble cependant marquée à la fois par une intensification de la fréquence de ces contrôles et par un accroissement de leurs enjeux. 
Le contrôle de l'activité enseignante peut également se réaliser dans le cadre d'auto-évaluations. Cette forme de responsabilisation s'est développée depuis le milieu des années 1990. Elle peut être conçue comme une première étape dans l'évaluation des enseignants et ne pas être accompagnée d'un contrôle externe. C'est le cas de l'Islande qui, en absence de tout mécanisme de contrôle des performances des écoles et des enseignants, a développé l'autoévaluation depuis 1995. La Hongrie, après avoir supprimé son inspection en 1985, a aussi introduit cette forme de responsabilisation à partir de 2000. Depuis 2007, un volet spécialement structuré autour du travail enseignant est inclus dans l'étude. À l'opposé, dans certains pays, l'auto-évaluation a été développée en complément d'une inspection externe déjà existante comme en République tchèque et en Estonie. En Slovénie, c'est l'évolution du rôle de l'inspection - depuis une fonction de contrôle de légalité vers un rôle d'animation des équipes pédagogiques - qui a conduit le ministère à développer un cadre d'auto-évaluation. Dans la très grande majorité des pays, le développement de ce mode de responsabilisation s'accompagne d'une structuration des critères de contrôle ou d'une vérification, par un agent externe à l'établissement scolaire, des critères choisis par les écoles.

L'évaluation de l'enseignant - cette fois-ci à titre individuel - peut être également menée en interne par l'interlocuteur de proximité, à savoir le chef d'établissement. C'est le cas, par exemple, en Autriche, en Belgique flamande, en République tchèque, en Grèce, en Lettonie, en Lituanie et en Slovénie. Dans certains pays, la décision peut être collective, parce que le conseil d'école y est associé, comme en Lituanie, ou parce qu'un interlocuteur extérieur à l'école intervient, comme en Grèce le conseiller scolaire.

Toujours en interne, l'évaluation peut être menée ou aidée par les pairs. Cette forme de responsabilisation reste aujourd'hui encore peu développée. Elle résulte le plus souvent de l'organisation de la conception des contenus d'enseignement dans un cadre d'autonomie, qui entraîne mécaniquement un travail en équipe, conduisant à son tour, à un contrôle par les pairs. Dans certains pays, cependant, cette évaluation peut être explicite. C'est le cas par exemple en Grèce, où le conseiller scolaire, qui est en charge, entre autres, des inspections individuelles, recueille également les avis des enseignants de la même discipline sur leur pair. De même, en Slovénie, le conseil des enseignants de l'établissement doit approuver par un vote secret à la majorité absolue les suggestions de promotion que le chef d'établissement présente au ministère de l'Éducation pour aval final.

Enfin, le milieu de la décennie 2000 est marqué par l'apparition de nouveaux mécanismes d'évaluation des performances individuelles des enseignants. Ces modalités de contrôle à forts enjeux sont associées à la fois à des incitations financières et à l'organisation de carrières différenciées. À travers le plus souvent des entretiens individualisés, elles visent à évaluer l'enseignant sur 
une base personnelle à l'aune d'un spectre large de critères : performances de ses élèves, mais aussi activités au sein de l'école ou dans un territoire plus large. Cette nouvelle forme de responsabilisation est déjà mise en œuvre en Belgique flamande, au Portugal ou en Angleterre et en cours de négociation en Espagne.

Enfin, il est à noter que dans notre recherche, à l'exception de la Belgique flamande, aucun pays n'a souligné l'existence d'une évaluation individuelle externe des enseignants par le grand public. Si les évaluations collectives sont dans certains pays rendues publiques, elles ne mentionnent pas de façon explicite les enseignants qui pourraient être jugés défaillants.

Ces différentes modalités d'évaluation tendent de plus en plus à se croiser, créant dans certains pays des réseaux d'évaluation internes et externes, individuelles et collectives. En effet, si traditionnellement, l'inspection individuelle était le mode quasi unique de contrôle du travail enseignant, aujourd'hui, les modalités multiples d'évaluation, décrites précédemment, tendent à se superposer.

C'est le cas de l'Autriche qui s'est longtemps cantonnée à une évaluation traditionnelle par l'inspection et par le chef d'établissement (qui est le supérieur direct de l'enseignant) et qui développe depuis 2006 un cadre national pour l'auto-évaluation. De la même façon, en République tchèque, le contrôle collectif et individuel par l'inspection et par le chef d'établissement se double depuis 2005 d'une auto-évaluation. L'Écosse est aussi caractéristique du développement de ces réseaux d'évaluations. Le contrôle des écoles par l'inspection (HMIE) est associé à une auto-évaluation qui suit la structure standardisée des Quality indicators ainsi qu'à des entretiens individuels.

Ces contrôles multiples permettent à la fois une analyse des processus du travail enseignant et une évaluation par les résultats.

\section{LA MONTÉE EN CHARGE DE L'ÉVALUATION PAR LES RÉSULTATS}

Traditionnellement, l'évaluation individuelle par l'inspection s'imposait surtout comme un contrôle des processus. Aujourd'hui, les nouvelles modalités de responsabilisation mettent l'accent sur les résultats, l'autonomie et la décentralisation croissantes permettant de moins en moins, par définition, d'imposer des normes nationales ou régionales.

Ainsi, en Écosse, l'évaluation interne vise-t-elle, entre autres, à appréhender l'écart qui existe entre un référentiel présenté sous forme d'indicateurs de qualité et les performances de l'école, dont le travail enseignant est considéré comme un des constituants principaux. En particulier, les résultats aux examens sont analysés dans le cadre des indicateurs de qualité "How good is my school?». De même, en République tchèque, en Estonie, en Hongrie ou en Suède, l'autoévaluation inclut-elle l'analyse des performances des enseignants. Dans de nombreux pays comme l'Autriche, la République tchèque, l'Espagne ou le 
Royaume-Uni, les inspections externes, de plus en plus structurées autour de normes standardisées, intègrent également une analyse en termes de résultat du travail enseignant. Que ce soit dans le cadre des évaluations internes ou externes, les résultats des élèves aux évaluations standardisées nationales deviennent de plus en plus un critère de jugement de la performance de l'école, et donc de l'équipe pédagogique, comme en Estonie, au Lichtenstein, à Malte, en Suède ou en Écosse.

De la même façon, se développent également depuis le milieu de la décennie 2000 des politiques d'évaluation individuelle des enseignants strictement fondées sur l'étude de leurs résultats personnels. Ces évaluations croisent un ensemble large de critères : depuis les performances académiques des élèves jusqu'à la participation à des activités de formation ou de recherche dans le cadre de projets pilotes, en passant par toute la gamme d'implications possibles au sein de l'école (soutien aux élèves, encadrement des nouveaux enseignants...).

Si l'évaluation par les résultats tend sinon à remplacer, du moins à fortement compléter le contrôle des processus et le respect des normes édictées nationalement ou localement, l'évolution est moins nette en ce qui concerne la dimension collective ou individuelle du contrôle du travail enseignant.

\section{UNE ÉVALUATION QUI OSCILLE ENTRE LE COLLECTIF ET L'INDIVIDUEL}

Les années 1990 ont semblé marquer la fin de l'évaluation individuelle des enseignants, le développement de l'autonomie scolaire permettant de faire apparaître un nouvel acteur collectif disposant de marges de manœuvre et donc évaluable en tant que tel : l'établissement scolaire. Le contrôle individuel a donc progressivement évolué vers une évaluation de l'équipe pédagogique. Ainsi, certains pays comme la Belgique flamande, la Roumanie ou très récemment, en 2007, la Belgique francophone, ont vu disparaître les inspections individuelles au profit des évaluations de l'équipe enseignante. L'Angleterre ou l'Écosse appartiennent historiquement à cette famille. Certains pays qui ont, eux, récemment développé leur inspection ou des formules d'auto-évaluation, comme l'Estonie ou Malte, ont directement mis en place un modèle de contrôle collectif. Enfin, d'autres pays comme la France tente de développer, dans un cadre qui n'est pas encore standardisé ni coordonné, une double évaluation individuelle et collective.

Alors qu'elles étaient devenues largement minoritaires dans ce contexte de collectivisation de la responsabilisation, les évaluations individuelles tendent de nouveau à se développer depuis le milieu de la décennie 2000. Ainsi, en Belgique flamande, depuis 2007-2008 (2008-2009 pour le primaire), les enseignants sont-ils soumis à un entretien d'évaluation de leurs performances renouvelé tous les trois ans. Deux jugements négatifs consécutifs peuvent conduire à un licenciement, comme pour tout fonctionnaire. En Espagne, le ministère de 
l'Éducation négocie actuellement un nouveau statut des enseignants non universitaires qui prévoit une carrière différenciée selon les performances individuelles ainsi qu'un «bonus de performance». Depuis 2007, le Portugal a aussi développé une évaluation individuelle qui est en lien avec des rémunérations complémentaires et des progressions différenciées de carrière - la carrière enseignante portugaise comprend désormais deux grandes étapes, correspondant à deux catégories : l'enseignant de base et l'enseignant expérimenté.

En Angleterre, les performance management arrangements, aux côtés d'autres mécanismes d'évaluation des résultats collectifs de l'école, se focalisent sur les performances individuelles des enseignants. Le chef d'établissement ou le responsable pédagogique de la discipline au sein de l'établissement sont responsables de l'entretien. L'Irlande du Nord et le Pays de Galles suivent des organisations similaires. Depuis 2007, l'Angleterre a également introduit des professional standards pour les enseignants, qui permettent d'évaluer si l'enseignant dispose des compétences nécessaires pour progresser dans la carrière. Ces évaluations ne sont pas rendues publiques.

Ce mouvement de responsabilisation individuelle vient renforcer la famille des pays qui, de façon privilégiée, avaient conservé une évaluation de l'enseignant par le chef d'établissement comme en République tchèque, en Lettonie, en Lituanie ou en Slovénie. Au total, à travers ce croisement croissant des contrôles sur l'individu et sur l'équipe, semble se dessiner un double mouvement de responsabilisation de l'enseignant : il est de plus en plus jugé à la fois à titre individuel en tant que responsable d'une classe et comme partie prenante d'une équipe pédagogique.

\section{DES RESPONSABILITÉS ÉLARGIES MAIS PEU DE MESURES INCITATIVES}

Si les attributions et la responsabilisation des enseignants se sont fortement développées depuis deux décennies, ces mutations ne se sont pas accompagnées de mesures incitatives dans tous les pays européens. On observe, au contraire, le phénomène inverse : que ce soit sous forme de rémunération additionnelle, d'allègement de cours ou de promotion, rares sont les pays qui ont prévu de "récompenser» les enseignants qui rempliraient avec efficacité leurs nouvelles attributions.

Les pays - une minorité - qui, dès les années 1980-1990, avaient prévu de gratifier les enseignants dans un contexte de développement de leurs attributions présentent trois caractéristiques : ils ont développé le plus souvent une évaluation centrée sur le chef d'établissement; ils se recrutent dans les pays de l'Europe centrale; ils proposent principalement un intéressement financier. Ainsi, en Hongrie, le chef d'établissement pratique un contrôle sur la qualité et la quantité de travail des enseignants qu'il encadre et peut octroyer à la fois des 
bonus exceptionnels ou des primes régulières. Depuis 2007, les résultats des élèves de l'enseignant aux épreuves standardisées peuvent également donner lieu à un bonus supplémentaire. En République tchèque, le chef d'établissement peut également, en liaison avec le conseil d'école, récompenser les enseignants pour des services supplémentaires ou un travail pédagogique de qualité. Ces revenus additionnels peuvent représenter jusqu'à $50 \%$ du salaire de base. La Slovaquie prévoit également la possibilité d'une rémunération supplémentaire pour les enseignants méritants. L'évaluation couvre un large spectre de critères, depuis les résultats académiques de la classe jusqu'à la participation à des activités pédagogiques régionales, en passant par des services et actions développées au sein de l'établissement. La Roumanie et la Lettonie distribuent également des primes aux enseignants.

À partir des années 2000, la mise en place de mesures incitatives s'est accélérée. Aujourd'hui, elles présentent deux formes principales. Les bénéfices des mutations du métier d'enseignant peuvent tout d'abord être collectifs. La reconnaissance d'un accroissement des tâches a ainsi conduit, souvent sous l'impulsion des syndicats, à une clarification des statuts et des missions ainsi qu'à des plans d'augmentation des salaires ou à des réévaluations des grilles de rémunération. Ainsi, en Écosse, le Teacher's agreement de 2001 s'est traduit, entre autres, par une forte augmentation des salaires (23\%), une révision des échelles de rémunération, un élargissement des opportunités de formation continue, davantage de libertés dans les obligations de service et un nombre d'heures de contact avec les élèves réduit. À Malte, l'élargissement du périmètre d'activités des enseignants a conduit là aussi à des hausses salariales générales et, dans le primaire, à l'affectation d'une heure et demie au développement des programmes scolaires de l'école. En Lituanie également, les syndicats ont négocié à la fois une clarification des conditions de travail et un plan pluriannuel d'augmentations salariales.

Aux côtés de ces réponses collectives, d'autres pays, comme nous l'avons vu précédemment, développent davantage des mesures incitatives individuelles. Ainsi, en Espagne, la nouvelle Loi sur l'éducation de 2006 (LOE) prévoit que l'évaluation des enseignants doit désormais entrer en ligne de compte dans l'attribution des primes financières et dans les promotions. Au Portugal, des évaluations positives permettent à la fois d'obtenir des bonus et d'accélérer la progression dans la carrière enseignante. La Lettonie a créée depuis 2007 un système de carrières différenciées qui, pour l'instant, fonctionne sur la base du volontariat. C'est l'enseignant qui choisit d'être évalué sur les critères de son choix parmi les dix-neuf propositions offertes, en apportant des éléments concrets sur ces sujets. Ces nouvelles configurations incitatives se traduisent par des salaires et des opportunités de carrière différenciées. 
En conclusion, si l'évaluation n'a pas accompagné, dans un ensemble légal cohérent, l'autonomie et l'élargissement du périmètre d'activité des enseignants, on a assisté cependant au développement progressif des mécanismes de contrôle. Dans des pays de plus en plus nombreux, ils portent à la fois sur le professionnel à titre individuel et sur son équipe pédagogique, sur la réalité de son activité, son adéquation aux normes imposées et ses performances. Dans une logique de New Public management, ce croisement d'évaluations multiples a fait progresser la reddition des comptes qui s'impose désormais aux enseignants qui ne sont plus totalement seuls et pilotes dans leur classe. Le développement récent d'évaluations individuelles à forts enjeux en termes de rémunération, de promotion et de conditions de travail (réduction des heures d'enseignement...) constitue une nouvelle étape dans le renforcement du contrôle du travail enseignant.

\section{BIBLIOGRAPHIE}

BROADFOOT P. (2000): «Un nouveau mode de régulation dans un système décentralisé : l'État-évaluateur», Revue française de pédagogie, 130, p. 43-55.

EURYDICE (2008) : Responsabilités et autonomie des enseignants, Bruxelles, 2008. MAROY C. (2005): «Les évolutions du travail enseignant en Europe. Facteurs de changement, incidences et résistances», Université Catholique de Louvain, Cahiers $d u$ GIRSEF, $\mathrm{n}^{\circ} 42$.

NIEVE G. (1988) : «On the cultivation of quality, efficiency and enterprise: an overview of recent trends in higher education in Western Europe, 1986-1988 ", European Journal of Education, 23 (1-2), p. 7-23. 
